УДК 620.98: 519.816

\title{
ВЫБОР ТОПЛИВА ДЛЯ ТЕПЛОВЫХ ЭЛЕКТРОСТАНЦИЙ В РАЙОНАХ НОВОГО ОСВОЕНИЯ С УЧЕТОМ ФАКТОРА НЕОПРЕДЕЛЕННОСТИ
}

\author{
Шакиров Владислав Альбертович, \\ mynovember@mail.ru
}

Институт систем энергетики им. Л.А. Мелентьева СО РАН, Россия, 664033, г. Иркутск, ул. Лермонтова, 130.

\begin{abstract}
Актуальность исследования обусловлена трансформацией методологии обоснования развития энергетики от иентрализованного государственного планирования к новой парадигме многостороннего процесса принятия решений и создания механизмов их реализации в условиях многокритериальности, множественности несовпадающих интересов, неопределенности исходной информации и условий дальнейшего развития. Новые условия обоснования решений исследуются в разрезе строительства тепловых электростанций в удаленных районах нового освоения, где в качестве топлива рассматриваются местные энергоресурсы. Выбор топлива определяет экономические и технические показатели электростанций, их экологические и социальные воздействия. Для обоснования выбора топлива тепловых электростанций с позиций многочисленных критериев в условиях неопределенности исходной информации и будущих условий развития необходимо создание новых методических подходов.

Цель: предложить методику многокритериального выбора топлива тепловой электростанции в районах нового освоения с учетом фактора неопределенности.

объекты: месторождения органического топлива в удаленных районах нового освоения.

Методы: мультипликативный метод анализа иерархий, метод многокритериальной теории полезности, методы интервального анализа, методы теории нечетких множеств.

Результаты. Проведен обзор современных методических подходов в задачах обоснования выбора топлива для электростанций. Предложена модификация метода мультипликативного анализа иерархий, которая позволяет учесть неопределенность исходной информации и неоднозначность предпочтений лица, принимающего решения, а также существенно снизить количество запросов информации. На основе модифицированного метода разработана методика многокритериального выбора топлива для тепловых электростанций в районах нового освоения. Ее применение рассмотрено на проблеме выбора угля в трех пунктах перспективного размещения тепловой электростанции в Омсукчанском и Северо-Эвенском районах Магаданской области. В качестве критериев сравнения альтернатив используются: стоимость топлива, ущерб от выброса загрязняющих веществ при сжигании топлива, обеспеченность запасами, условия освоения и добычи, воздействие выбросов при сжигании топлива на здоровье населения, обеспечение занятости местного населения.
\end{abstract}

\section{Ключевые слова:}

Тепловые электростанции, топливо, уголь, многокритериальный выбор, неопределенность исходной информации.

\section{Введение}

При выборе пункта размещения тепловой электростанции (ТЭС) на стадии технико-экономического обоснования вариантно рассматривается наиболее эффективный вид топлива, способы его подачи на электростанцию. В период существования жесткой централизованной системы планирования развития энергетических систем такие задачи входили в общую иерархию и решались с использованием оптимизационных моделей в несколько итераций с рассмотрением на уровнях единой энергосистемы, объединенных и районных энергосистем. Так, с учетом результатов прогнозирования нагрузок и электропотребления, в рамках задачи разработки топливноэнергетического баланса, определялись размеры выделяемых топливных ресурсов, проводилась их экономическая оценка, намечались варианты возможной структуры генерирующих мощностей. Далее решение по структуре генерирующих мощностей уточнялось, проводилась оптимизация мощностей электростанций, определялись ориентировочные сроки ввода, пункты и площадки. Основным критерием оценки была народнохозяйственная эффективность с минимумом приведенных затрат при выполнении ряда технических и экологических ограничений [1].
Проводимые с начала 90-х годов прошлого века рыночные реформы изменили условия развития энергетических систем. Функция государства теперь заключается в выработке и проведении общей энергетической политики. Увеличилось число лиц, принимающих решения, большую роль стали играть региональные органы управления и владельцы энергетических объектов, которые оказывают влияние на их функционирование и развитие [1].

В результате реформирования энергетики произошла трансформация методологии обоснования ее развития от централизованного государственного планирования к новой парадигме многостороннего процесса обоснования решений и создания механизмов их реализации в условиях многокритериальности, множественности несовпадающих интересов, неопределенности исходной информации и условий дальнейшего развития [1].

В исследованиях можно выделить три группы задач, связанных с выбором топлива для электростанций. Первая группа задач связана с оценкой в масштабах региона или страны только экологических последствий ввода станций на различных видах топлива за период жизненного цикла, от этапа строительства до этапа ликвидации [2]. Вторая группа за- 
дач связана с выбором перспективных для исследуемого района технологий производства энергии по укрупненным показателям стоимости, экологических и социальных воздействий $[1,3-5]$. Состав альтернатив определяется имеющимися в районе традиционными органическими и возобновляемыми энергоресурсами.

Наиболее детально проблема выбора топлива рассматривается в третьей группе задач, связанных с выбором месторождения или поставщика определенного вида топлива на этапе проектирования электростанции. Сравнение проводится по стоимости топлива, условиям добычи и транспортировки, характеристикам топлива (теплоте сгорания, влажности, зольности, содержанию азота, серы и т. п.), величине запасов и другим. Для решения задач этой группы используется два основных подхода. Подход на основе оценки экономической эффективности заключается в сравнении вариантов по таким показателям, как чистый дисконтированный доход, приведенные затраты, нормированная стоимость производства энергии $[1,6]$. Неэкономические факторы или учитываются в виде ограничений, или приводятся с использованием различных допущений в денежное выражение для возможности учета в экономической оценке.

Ввиду тенденций повышения значимости экологических и социальных факторов расширяется применение многокритериального подхода к выбору топлива для ТЭС. Наиболее часто применяются методы TOPSIS, PROMETHEE, ELECTRE, AHP (Analytic Hierarchy Process) [7-10]. В основе подхода лежит раздельное рассмотрение экономического и неэкономических факторов с позиций их относительной важности для лица или группы лиц, принимающих решения (ЛПР). Многокритериальный подход имеет преимущество, когда альтернативные варианты имеют существенные различия в экологических или социальных воздействиях, а также в случаях, если альтернативные варианты обладают практически равными экономическими показателями. В таких ситуациях появляется необходимость достижения по неэкономическим критериям лучших возможных значений.

Для учета неточности, связанной с субъективным характером оценок экспертов, расхождением оценок нескольких экспертов, неопределенности исходной информации необходимо развитие методов многокритериального выбора. В статье предлагается модификация многокритериального метода АНР для выбора топлива ТЭС с учетом неопределенности исходной информации и предпочтений лиц, принимающих решения.

\section{Постановка проблемы многокритериального выбора топлива}

При рассмотрении потенциальных пунктов строительства ТЭС в зависимости от ресурсного потенциала и транспортной инфраструктуры набор альтернатив может включать один или несколько видов топлива разных месторождений.

Выбор альтернативы влечет длительные экономические и социальные последствия, воздействия на окружающую среду, здоровье и безопасность населения. Поэтому при принятии решений преследуются многочисленные цели, направленные на обеспечение высокой экономической эффективности и минимизацию негативных последствий, связанных с выбором топлива. Для количественного измерения степени достижения целей формулируются критерии. Например, в качестве критериев могут выступать: стоимость топлива с учетом доставки; экспертная оценка условий освоения месторождений, добычи и транспорта энергоресурса; выброс вредных веществ в атмосферу при использовании топлива; изменение продолжительности жизни местного населения из-за вредных выбросов ТЭС при использовании топлива.

Точная количественная оценка многих критериев затруднена вследствие неполноты и неопределенности исходной информации. Предпочтения ЛПР также неоднозначны из-за неопределенности будущих условий.

Задача многокритериального выбора сформулирована следующим образом: пусть имеется $m$ альтернатив $A=\left\{a_{1}, a_{2}, \ldots, a_{m}\right\}$ топлива для ТЭС. Последствия, связанные с выбором альтернативы, характеризуются в общем случае нечеткими оценками $\left\{\tilde{v}_{1}, \tilde{v}_{2}, \ldots, \tilde{v}_{m}\right\}$ по $n$ критериям $F=\left\{f_{1}, f_{2}, \ldots, f_{n}\right\}$. Требуется упорядочить альтернативы множества $A$ с учетом нескольких вариантов предпочтений ЛПР, отвечающих возможным сценариям перспективного развития.

Для сформулированной задачи многокритериального выбора топлива целесообразно применение метода, ориентированного на непосредственное сравнение малого числа заданных альтернатив. Наиболее эффективным из таких методов является АНР. Широкой апробации АНР способствовали удобство для ЛПР парных сравнений альтернатив и критериев с использованием специальной шкалы оценок. В связи с критикой метода АНР по ряду положений были разработаны его различные модификации [11]. Одной из наиболее известных является мультипликативная версия метода АНР, предложенная Ф. Лутсмой.

\section{Основные этапы метода мультипликативного АНР}

Метод мультипликативного АНР включает следующие основные этапы [12]:

1. Структуризация и выделение базовых элементов проблемы: цели, критерии для оценки степени достижения целей $f_{1}, f_{2}, \ldots, f_{n}$ и альтернативы $a_{1}, \ldots a_{m}$.

2. Оценка альтернатив по критериям с использованием формализованных моделей, данных объектов-аналогов или знаний экспертов.

3. Парные сравнения критериев и альтернатив по отдельным критериям с заполнением матриц парных сравнений элементами:

$$
d_{i j}=h^{\delta_{i j}},
$$

где $h$-параметр шкалы, равен 2 при заполнении матриц парных сравнений альтернатив, $2^{0,5}-$ при заполнении матриц парных сравнений критериев [12]; $\delta_{i j}-$ числовая оценка сравнительной предпочтительности $i$-го и $j$-го элементов от 0 до 8. Оценка сравнительной 
предпочтительности 0 соответствует безразличию между сравниваемыми элементами; 2 - слабому превосходству $i$-го элемента на $j$-м элементом; 4 - определенному превосходству $i$-го элемента на $j$-м элементом; 6 - сильному превосходству $i$-го элемента на $j$-м элементом; 8 - очень сильному превосходству $i$-го элемента на $j$-м элементом.

Частная ценность $s_{i q}$ альтернативы $a_{i}$ по $q$-му критерию определяется [12]:

$$
s_{i q}=\left(\prod_{j=1}^{m} d_{i j q}\right)^{\frac{1}{m}} .
$$

Важность $q$-го критерия рассчитывается по формуле [12]:

$$
\omega_{q}=\left(\prod_{l=1}^{n} 2^{0,5 \delta_{q l}}\right)^{\frac{1}{n}} .
$$

4. Определение многокритериальных оценок альтернатив путем мультипликативной свертки частных ценностей [12]:

$$
s\left(a_{i}\right)=\prod_{q=1}^{n} s_{i q}^{\omega_{q}} .
$$

\section{Модификация мультипликативного АНР для учета фактора неопределенности}

Для учета неопределенности, связанной с субъективным характером предпочтений ЛПР, Д. Чангом, Дж. Бакли были предложены нечеткие модификации метода АНР [13, 14]. В основе подходов лежит замена оценок парных сравнений $\delta_{i j}$ нечеткими числами, что требует многочисленных запросов к ЛПР и является весьма трудоемким. Повышается вероятность ошибочных сравнений и несогласованных ответов.

В этой связи в статье предлагается подход к учету неопределенности исходной информации и предпочтений ЛПР, направленный одновременно на снижение числа запросов к ЛПР. В основе подхода лежит предложенный Ф. Лутсмой способ определения $\delta_{i j}$ без участия ЛПР. Так, диапазон оценок по критерию $\left[c_{\min }, c_{\mathrm{max}}\right]$ может быть разбит на несколько интервалов (категорий). Оценка $\mu$ по этой шкале может быть определена по выражению [12]:

$$
\left\{\begin{array}{l}
\mu=\log _{2}\left(\frac{c-c_{\text {min }}}{c_{\text {max }}-c_{\text {min }}} \cdot 2^{k}\right), \text { если } v\left(c_{\text {min }}\right)>v\left(c_{\max }\right) ; \\
\mu=\log _{2}\left(\frac{c_{\text {max }}-c}{c_{\text {max }}-c_{\text {min }}} \cdot 2^{k}\right), \text { если } v\left(c_{\text {max }}\right)>v\left(c_{\text {min }}\right),
\end{array}\right.
$$

где $k$ - число категорий; $v\left(c_{\min }\right), v\left(c_{\max }\right)$ - ценность для ЛПР максимальной и минимальной оценок по критерию, $c$ - оценка по критерию.

Оценка шкалы сравнительной предпочтительности альтернатив $a_{i}, a_{j}$ определяется:

$$
\delta_{i j}=\mu_{j}-\mu_{i} .
$$

В результате использования (5) и (6) число запросов к ЛПР существенно сокращается.

Для учета особенностей предпочтений ЛПР и их неоднозначности предлагается дополнить мультипликативный АНР процедурой построения однокритериальных функций ценности интервального вида (рис. 1,a).

Худшей оценке по критерию $y_{q}^{0}$ присваивается ценность $v\left(y_{q}{ }^{0}\right)=0$, лучшей оценке по критерию $y_{q}{ }^{1}$ присваивается ценность $v\left(y_{q}{ }^{1}\right)=1$. Промежуточные оценки с ценностью $0,25,0,5$ и 0,75 должно назначить ЛПР с возможностью использования интервальных чисел [15]. Функции ценности позволяют получить оценки ценности любых альтернатив в области определения без дополнительных запросов к ЛПР (рис. 1, б).

Подстановка в (1), (5) и (6) значений функций ценности определяет оценку матрицы парных сравнений:

$$
d_{i j}=\frac{1-v_{q}\left(y_{q j}\right)}{1-v_{q}\left(y_{q i}\right)} .
$$
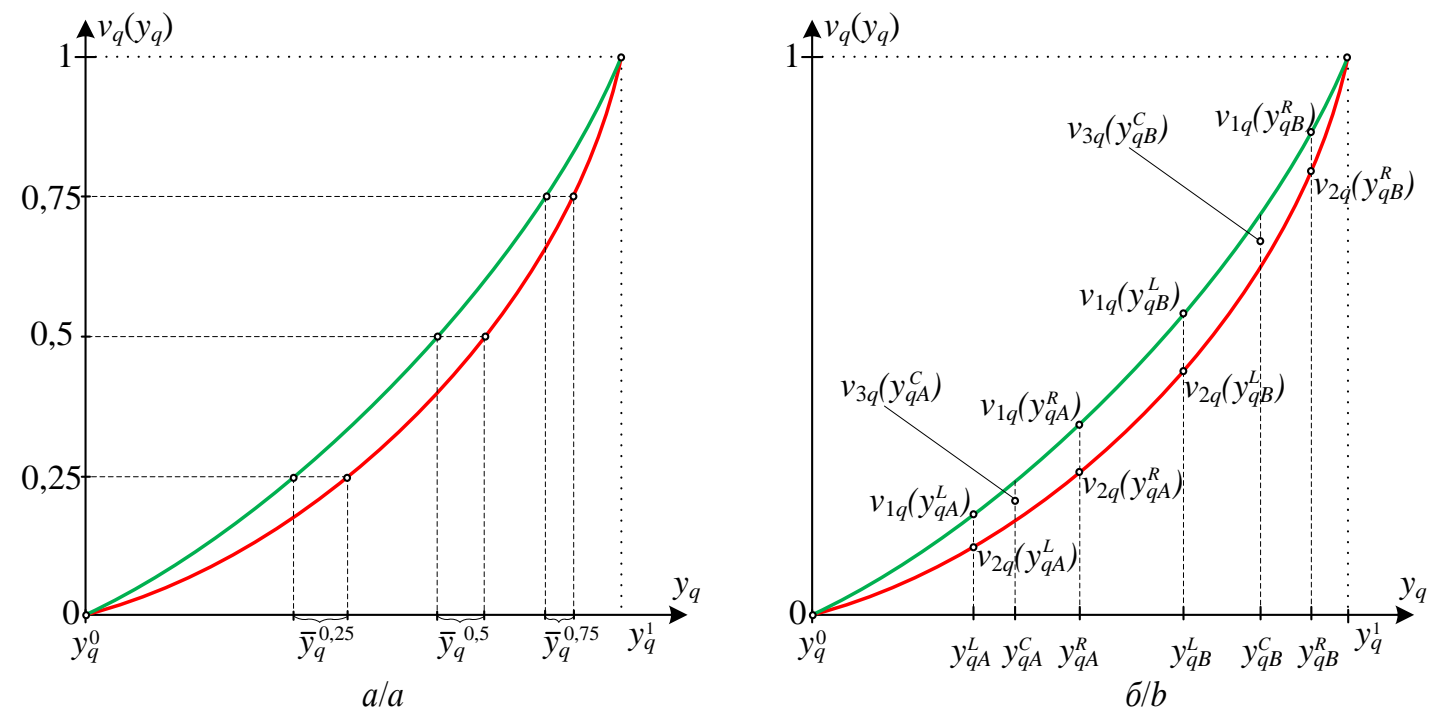

Pис. 1. Однокритериальные функции ценности интервального вида: а) процедура построения; б) определение значений функиии

Fig. 1. Single-criterion interval value function: a) construction procedure; b) definition of function values 
Так как в основе выражения (7) лежит формула (5) с операцией логарифмирования, то необходимо принять $v_{q}\left(y_{q}^{1}\right)=255 / 256$.

Определение элементов матриц парных сравнений по формуле (7) лежит в основе предлагаемой методики выбора топлива ТЭС с учетом фактора неопределенности. Голубым цветом на рис. 2 отмечены новые этапы, добавленные к методу мультипликативного AHP.

Рассмотрим отдельные этапы методики на примере сравнения трех альтернатив $a_{A}, a_{B}, a_{C}$.

На первом этапе с помощью ЛПР формируется иерархия, включающая три уровня: цели, $n$ критериев и $m$ альтернатив (видов топлива, месторождений).

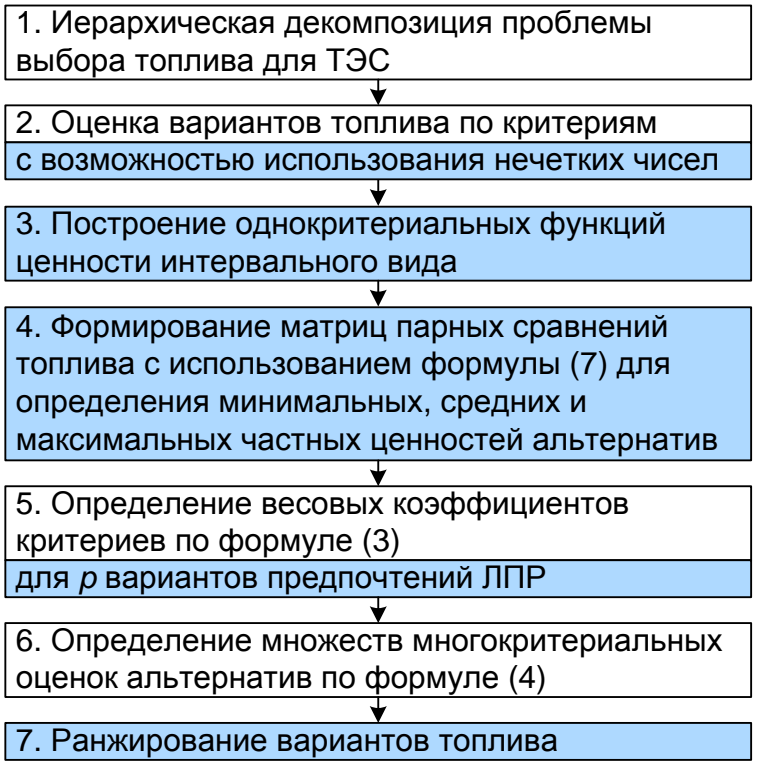

Рис. 2. Методика многокритериального выбора топлива для тепловой электростаниии на основе метода мультипликативного анализа иерархий с учетом фактора неопределенности

Fig. 2. Methodology of multi-criteria fuel selection for a thermal power plant based on the multiplicative analytic hierarchy process method taking into account the uncertainty factor

На втором этапе проводится оценка альтернатив по критериям. В условиях неопределенности исходной информации для этого удобно использовать нечеткие числа с треугольной функцией принадлежности $f_{\Delta}\left(y, y^{L}, y^{C}, y^{R}\right)$, а также интервалы $\left[y^{L}, y^{R}\right]$. Значения $y^{L}, y^{C}, y^{R}$ соответствуют худшей, наиболее достоверной (вероятной) и лучшей оценкам. Например, показатели качества угля, такие как теплота сгорания, содержание золы, серы и т. п., характеризуются интервалом возможных значений и средней оценкой для месторождения.

На третьем этапе проводится построение функций ценности интервального вида для каждого критерия. На рис. 1, $\sigma$ показан пример определения ценности альтернатив $a_{A}, a_{B}$. Определяются по два граничных значения ценности каждой оценки: $v_{1 q}\left(y_{q}^{L}\right), v_{2 q}\left(y_{q}^{L}\right)$ и $v_{1 q}\left(y_{q}^{R}\right), v_{2 q}\left(y_{q}^{R}\right)$, а также средняя ценность $v_{3 q}\left(y_{q}^{c}\right)$ альтернативы:

$$
v_{3 q}\left(y_{q}^{C}\right)=\frac{1}{2}\left[v_{1 q}\left(y_{q}^{C}\right)+v_{2 q}\left(y_{q}^{C}\right)\right] .
$$

На этапе 4 проводится формирование матриц парных сравнений альтернатив. Для того чтобы определить максимально и минимально возможные частные ценности при нечетких оценках альтернатив и интервальной функции ценности необходимо сформировать матрицы специальным образом. Так, для определения максимальной возможной частной ценности альтернативы в матрицу парных сравнений должны быть внесены наибольшие оценки сравнительной важности этой альтернативы и наименьшие - других альтернатив, и наоборот - для получения минимальной возможной частной ценности. Например, для альтернативы $a_{A}$ :

$$
\begin{aligned}
& D_{\max A}^{v_{1 q}}=\left|\begin{array}{l}
1 ; d_{\max A \min B}^{v_{1 q}} ; d_{\max A \min C}^{v_{1 q}} \\
d_{\min B \max A}^{v_{1 q}} ; 1 ; d_{\min B \min C}^{v_{1 q}} \\
d_{\min C \max A}^{v_{1 q}} ; d_{\min C \min B}^{v_{1 q}} ; 1
\end{array}\right|= \\
& \left.1 ; \quad\left(\frac{1-v_{1 q}\left(y_{q B}^{R}\right)}{1-v_{1 q}\left(y_{q A}^{L}\right)}\right) ;\left(\frac{1-v_{1 q}\left(y_{q C}^{R}\right)}{1-v_{1 q}\left(y_{q A}^{L}\right)}\right)\right) \\
& \left.=\left(\frac{1-v_{1 q}\left(y_{q A}^{L}\right)}{1-v_{1 q}\left(y_{q B}^{R}\right)}\right) ; \quad 1 ; \quad\left(\frac{\left.1-v_{1 q}\left(y_{q C}^{R}\right)\right)}{1-v_{1 q}\left(y_{q B}^{R}\right)}\right)\right) \text {, } \\
& \left(\frac{1-v_{1 q}\left(y_{q A}^{L}\right)}{1-v_{1 q}\left(y_{q C}^{R}\right)}\right) ;\left(\frac{1-v_{1 q}\left(y_{q B}^{R}\right)}{1-v_{1 q}\left(y_{q C}^{R}\right)}\right) ; \quad 1 ;
\end{aligned}
$$

где $D_{\max }^{v_{1 q}}-$ матрица парных сравнений альтернатив, сформированная с использованием функции ценности $v_{1 q}$ таким образом, чтобы определить максимальную возможную частную ценность альтернативы $a_{A}$; $d_{\max A \min B}^{v_{1 q}}, d_{\max A \min C}^{v_{1 q}}, d_{\min B \min C}^{v_{1 q}}-$ элементы матрицы парных сравнений, соответствующие оценкам сравнительной важности трех альтернатив, при которых обеспечивается максимально возможная частная ценность альтернативы $a_{A}$.

Далее по формулам, аналогичным (8), формируются матрицы $D_{\min A}^{v_{1 q}}, \quad D_{\max A}^{v_{2 q}}, \quad D_{\min A}^{v_{2 q}}, \quad D_{\max B}^{v_{1 q}}, \quad D_{\min B}^{v_{1 q}}$, $D_{\operatorname{maxB}}^{v_{2 q}}, \quad D_{\min B}^{v_{2 q}}, \quad D_{\max C}^{v_{1 q}}, D_{\min C}^{v_{1 q}}, \quad D_{\max C}^{v_{2 q}}, \quad D_{\min C}^{v_{2 q}}$, a также матрица $D_{\text {avg }}^{v_{3 q}}$ для определения средних частных ценностей альтернатив $a_{A}, a_{B}, a_{C}$ :

$$
\begin{aligned}
& D_{\text {avg }}^{v_{3 q}}=\left|\begin{array}{l}
1 ; d_{\text {avgAB }}^{v_{3 q}} ; d_{\text {avg }}^{v_{3 q C}} \\
d_{\text {avg } v_{3 q}}^{v_{3 q}} ; 1 ; d_{\text {avgBC }}^{v_{3 q}} \\
d_{\text {avgCA }}^{v_{3 q}} ; d_{\text {avg }}^{v_{3 q}} ; 1
\end{array}\right|= \\
& =\left|\begin{array}{cc}
1 ; & \left(\frac{1-v_{3 q}\left(y_{q B}^{C}\right)}{1-v_{3 q}\left(y_{q A}^{C}\right)}\right) ;\left(\frac{1-v_{3 q}\left(y_{q C}^{C}\right)}{1-v_{3 q}\left(y_{q A}^{C}\right)}\right) \\
\left(\frac{1-v_{3 q}\left(y_{q A}^{C}\right)}{1-v_{3 q}\left(y_{q B}^{C}\right)}\right) ; & 1 ; \quad\left(\frac{1-v_{3 q}\left(y_{q C}^{C}\right)}{1-v_{3 q}\left(y_{q B}^{C}\right)}\right) \\
\left(\frac{1-v_{3 q}\left(y_{q A}^{C}\right)}{1-v_{3 q}\left(y_{q C}^{C}\right)}\right) ;\left(\frac{1-v_{3 q}\left(y_{q B}^{C}\right)}{1-v_{3 q}\left(y_{q C}^{C}\right)}\right) ; 1 ; & 1 ;
\end{array}\right| .
\end{aligned}
$$


Сформированные матрицы парных сравнений позволяют с помощью формулы (2) определить максимальную, минимальную и среднюю оценки частной ценности альтернатив.

Например, расчет частных ценностей альтернатив $a_{A}, \quad a_{B}, \quad a_{C}$ по матрицам парных сравнений $D_{\max A}^{v_{1 q}} D_{\max A}^{v_{2 q}}$ проводится следующим образом:

$$
\begin{gathered}
S_{\mathrm{qmax} A}=\left(\max \left(d_{\max A \min B}^{v_{1 q}} \cdot d_{\max A \min C}^{v_{1 q}} ; d_{\max A \min B}^{v_{2 q}} \cdot d_{\max A \operatorname{minC}}^{v_{2 q}}\right)\right)^{\frac{1}{m}} ; \\
S_{\mathrm{qB} \max A}=\left(d_{\operatorname{minBmax} A}^{v_{i q}} \cdot d_{\operatorname{minB\operatorname {minC}}}^{v_{i q}}\right)^{\frac{1}{m}} ; \\
s_{q C \max A}=\left(d_{\min C \max A}^{v_{i q}} \cdot d_{\min C \min }^{v_{i q}}\right)^{\frac{1}{m}},
\end{gathered}
$$

где

- $i=1$, если $d_{\max A \min B}^{v_{1 q}} \cdot d_{\max A \min C}^{v_{1 q}} \geq d_{\max A \min B}^{v_{2 q}} \cdot d_{\max A \operatorname{minC}}^{v_{2 q}}$;

- $i=2$, если $d_{\max A \min B}^{v_{1 q}} \cdot d_{\max A \min C}^{v_{1 q}}<d_{\max A \min B}^{v_{2 q}} \cdot d_{\max A \min C}^{v_{2 q}}$.

Частные ценности альтернатив $a_{A}, a_{B}, a_{C}$ при оценке по матрице парных сравнений $D_{\text {avg }}^{v_{3 q}}$ :

$$
\begin{aligned}
& s_{\text {qavgA }}=\left(d_{\text {avgAB }}^{v_{3 q}} \cdot d_{\text {avgAC }}^{v_{3 q}}\right)^{\frac{1}{m}}, \\
& s_{q a v g B}=\left(d_{\text {avgBA }}^{v_{3 q}} \cdot d_{\text {avg } v^{v_{3 q}}}\right)^{\frac{1}{m}}, \\
& s_{\text {qavgC }}=\left(d_{\text {avgCA }}^{v_{3 q}} \cdot d_{\text {avgCB}}^{v_{3 q}}\right)^{\frac{1}{m}} .
\end{aligned}
$$

На пятом этапе проводится оценка весовых коэффициентов критериев. ЛПР может сформировать несколько матриц парных сравнений критериев, отражающих различные предпочтения с учетом возможных вариантов развития рассматриваемых районов размещения ТЭС. Таким образом, с помощью формулы (3) проводится оценка $p$ множеств коэффициентов важности:

$$
\begin{gathered}
W=\left\{W_{1}, W_{2}, \ldots, W_{p}\right\}= \\
=\left\{\left\{\omega_{11}, \omega_{21}, \ldots, \omega_{n 1}\right\}, \ldots,\left\{\omega_{1 p}, \omega_{2 p}, \ldots, \omega_{n p}\right\}\right\} .
\end{gathered}
$$

На шестом этапе проводится многокритериальная оценка альтернатив по формуле (4) с использованием множеств оценок частной ценности и множеств коэффициентов важности. В целом формируется $2 m p+p$ множеств многокритериальных оценок. Для удобства сопоставления полученных результатов оценки нормируются.

На рис. 3 представлен пример полученных многокритериальных оценок трех альтернатив.

Максимальные $s_{\text {maxi }}\left(a_{i}\right)$, минимальные $s_{\operatorname{mini}}\left(a_{i}\right)$, а также средние $s_{\text {avgmin }}\left(a_{i}\right), \mathrm{s}_{\text {avgmax }}\left(a_{i}\right)$ оценки определяются из множеств многокритериальных оценок, полученных с учетом неопределенности исходной информации и $p$ вариантов предпочтений ЛПР.

На седьмом этапе проводится ранжирование альтернатив. Из рис. 3 видно, что сравнение альтернатив может не требовать дополнительного анализа, как в случае с превосходством альтернативы $a_{C}$ над альтернативами $a_{A}$ и $a_{B}$. Однако может возникать ситуа- ция, когда потребуется количественная оценка превосходства одной альтернативы по отношению к другой, как, например, в случае с альтернативами $a_{A}$ и $a_{B}$. Для таких ситуаций предлагается использовать две модели сравнения альтернатив.

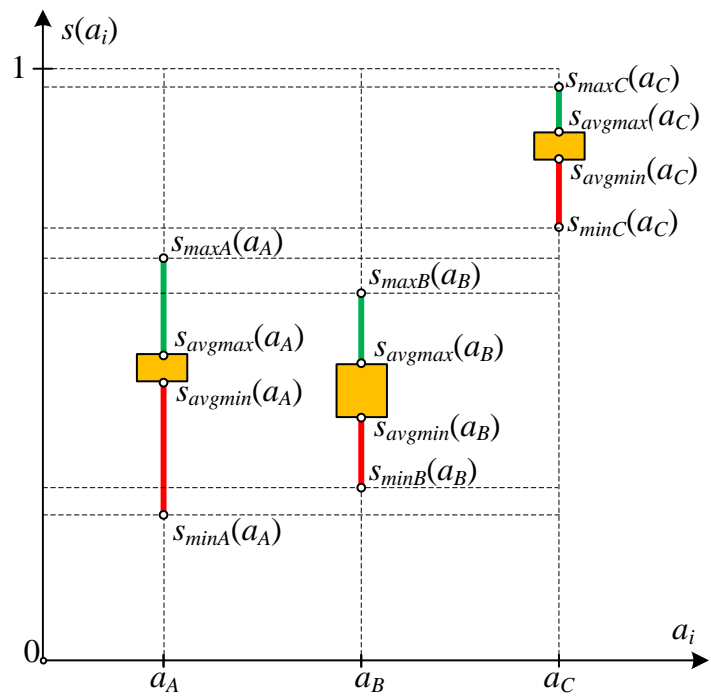

Puс. 3. Пример многокритериальных оценок альтернатив в условиях неопределенности исходной информачии и предпочтений лища, принимающего решения

Fig. 3. Example of multi-criteria assessments of alternatives in the face of uncertainty in the initial information and preferences of decision makers

1. Модель сравнения альтернатив по оценке относительного доминирования:

$$
F_{\text {domij }}=\frac{z_{i j}}{2 m p+p},
$$

где $z_{i j}$ - количество сравнений альтернатив $a_{i}$ и $a_{j}$, при которых многокритериальная оценка альтернативы $s_{t}\left(a_{i}\right)>s_{t}\left(a_{j}\right) ; t=1, \ldots, 2 m p+p$.

Если $F_{d o m i j}>0,5$, альтернатива $a_{i}$ предпочтительнее $a_{j}$.

2. Модель сравнения альтернатив на основе суммы разностей многокритериальных оценок:

$$
F_{s i j}=\frac{\sum_{t=1}^{2 m p+p} \max \left[s_{t}\left(a_{i}\right)-s_{t}\left(a_{j}\right) ; 0\right]}{\sum_{t=1}^{2 m p+p}\left|s_{t}\left(a_{i}\right)-s_{t}\left(a_{j}\right)\right|} .
$$

Если $F_{s i j}>0,5$, альтернатива $a_{i}$ предпочтительнее $a_{j}$. Окончательное решение ЛПР или группа ЛПР принимает исходя из графического представления многокритериальных оценок и полученных количественных показателей.

Применение методики выбора топлива для тепловой электростанции в Магаданской области

Магаданская область является одним из ключевых субъектов Российской Федерации с богатейшей минерально-сырьевой базой. На территории региона имеются разведанные запасы каменных и бурых углей, золота и серебра, олова, вольфрама, свинца, цин- 
ка, стройматериалов, пресных подземных и минеральных вод. Значительную перспективу имеют проекты горнодобывающей отрасли Омсукчанского и Северо-Эвенского районов. Сдерживающий фактор освоения новых месторождений связан с их большой удаленностью от объектов энергетической и транспортной инфраструктуры. Реализация крупных проектов потребует создание в непосредственной близости источника электрической и тепловой энергии.

На рис. 4 представлен ситуационный план с намеченными перспективными пунктами П1, П2, П3 строительства ТЭС. Перспектива развития в пунктах определяется следующими проектами:

П1: Омсукчанский угольный кластер; морской порт с угольным терминалом в бухте Пестрая Дресва; Дукатский хаб, включающий месторождения серебра Дукат, Лунное, Гольцовое, Перевальное; Верхнеомолонское месторождение молибдена; оловорудные месторождения.

П2: Ольдянинский рудный узел с общим прогнозным ресурсным потенциалом 568 тыс. т молибдена, 379 тыс. т меди, 20,8 т золота, 19 т рения; ЮжноОмолонский железорудный узел с общим ресурсным потенциалом железной руды 1990 млн т.

П3: месторождения россыпного золота, рудного золота и серебра, коренные месторождения золота и серебра; запасы яшмы, берилла, пренита, янтаря; рыбоперерабатывающий завод; завод по глубокой переработке продукции оленеводства; мясомолочный комбинат; птицефабрика; морской порт.
Характеристика пунктов и месторождений угля дана в табл. 1. В табл. 2 представлена краткая характеристика угля [16-18].

В соответствии с методикой многокритериального выбора топлива (рис. 2) на первом этапе формируется иерархия целей и критериев для оценки топлива. Сравнение топлива для ТЭС в пунктах П1 и П2 проводится с помощью множества критериев $F_{1}=\left\{f_{1}, f_{2}, f_{31}, f_{41}\right\}$, в пункте П3 - с помощью множества $F_{2}=\left\{f_{1}, f_{2}, f_{32}, f_{42}\right\}$.

Критерий $f_{1}-$ «Удельная стоимость угля в условном эквиваленте» служит для оценки достижения цели «Минимизация затрат на топливо».

Критерий $f_{2}-$ «Удельный ущерб от выбросов при сжигании топлива на ТЭС» используется для оценки достижения цели «Минимизация воздействия на окружающую среду».

Критерий $f_{31}$ - «Обеспеченность запасами угля в условном эквиваленте» используется для оценки достижения цели «Максимизация запасов угля».

Критерий $f_{41}-$ «Условия освоения месторождения, добычи и транспорта угля» используется для оценки достижения цели «Минимизация сложности добычи угля».

Критерий $f_{32}$ - «Изменение продолжительности жизни местного населения из-за вредных выбросов ТЭС» служит для оценки достижения цели «Минимизация воздействия на здоровье населения».

Критерий $f_{42}$ - «Обеспечение занятости местного населения» используется для учета социального фактора при принятии решений.

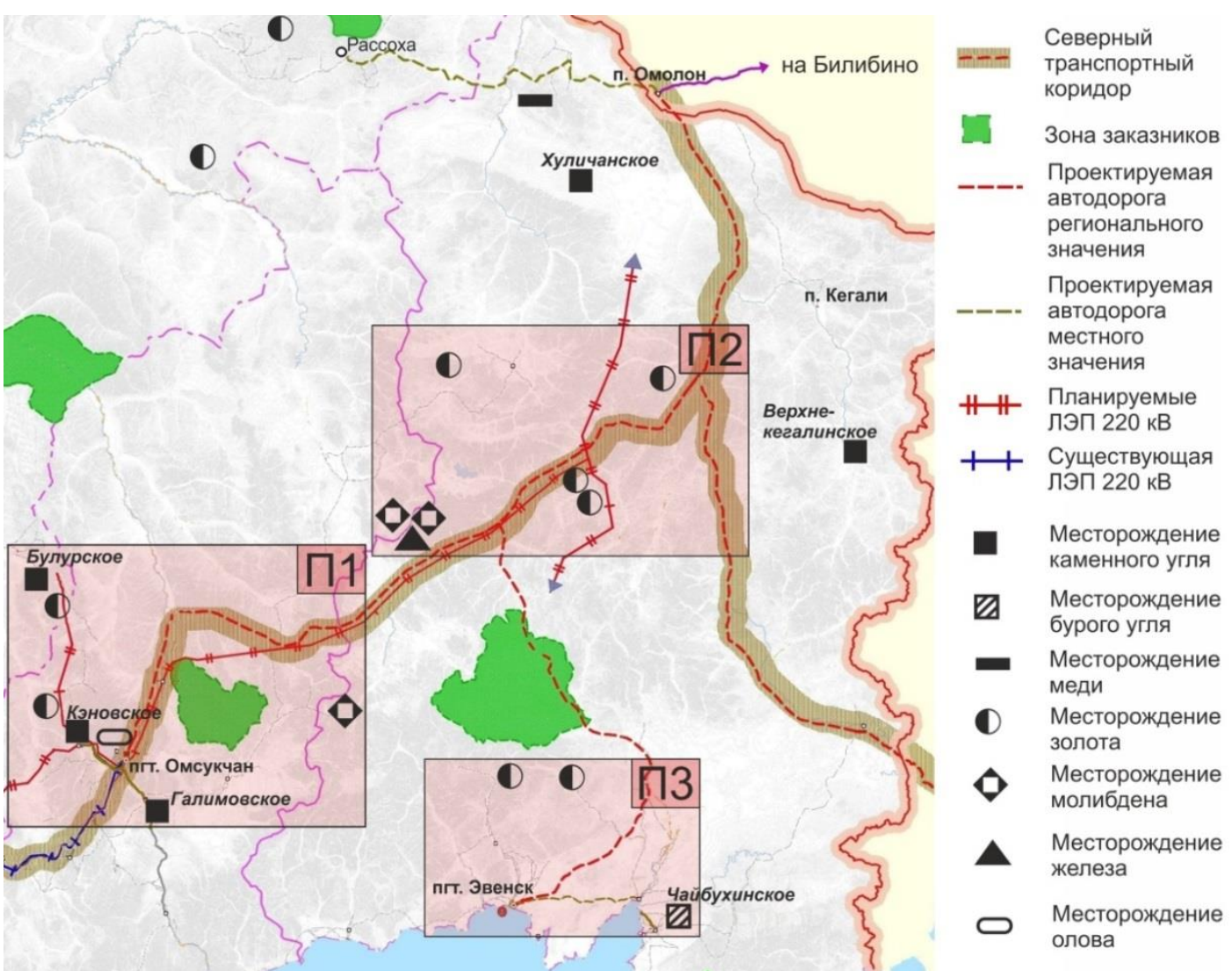

Рис. 4. Ситуационный план рассматриваемой территории

Fig. 4. Situation plan of the territory 
Таблица 1. Характеристика месторождений угля для топливообеспечения тепловых электростанций

Table 1. Characteristics of coal deposits for fuel supply of thermal power plants

\begin{tabular}{|c|c|c|c|c|}
\hline $\begin{array}{c}\text { Пункт } \\
\text { Point }\end{array}$ & $\begin{array}{l}\text { Месторождения угля } \\
\text { Coal deposits }\end{array}$ & $\begin{array}{c}\text { Способ освоения } \\
\text { Coal deposit } \\
\text { development } \\
\text { method } \\
\end{array}$ & $\begin{array}{l}\text { Характеристика района } \\
\text { Characteristic of the area }\end{array}$ & $\begin{array}{l}\text { Мощность } \\
\text { пластов, м } \\
\text { Coal steam } \\
\text { thickness, } \mathrm{m}\end{array}$ \\
\hline \multirow{3}{*}{$\begin{array}{l}\Pi 1 \\
\mathrm{P} 1\end{array}$} & $\begin{array}{c}\text { Галимовское } \\
\text { месторождение каменного угля } \\
\text { Galimovskoe hard coal deposit }\end{array}$ & $\begin{array}{l}\text { Подземный } \\
\text { Underground }\end{array}$ & $\begin{array}{c}\text { Район освоен, } 22 \text { км до пгт. Омсукчан, } \\
\text { имеются дороги } \\
\text { The area is developed, } 22 \mathrm{~km} \text { to Omsukchan } \\
\text { settlement, transport infrastructure }\end{array}$ & $1,2-13$ \\
\hline & $\begin{array}{l}\text { Кэновское месторождение } \\
\text { каменного угля } \\
\text { Kenovskoe hard coal deposit }\end{array}$ & $\begin{array}{l}\text { Открытый } \\
\text { Surface }\end{array}$ & $\begin{array}{c}\text { Район относительно освоен, } \\
40 \text { км до п. Омсукчан, имеются дороги } \\
\text { The area is relatively developed, } \\
40 \mathrm{~km} \text { to Omsukchan, roads }\end{array}$ & $1-6$ \\
\hline & $\begin{array}{c}\text { Булурское месторождение } \\
\text { каменного угля } \\
\text { Bulurskoe hard coal deposit }\end{array}$ & $\begin{array}{c}\text { Открытый } \\
\text { и подземный } \\
\text { Surface and } \\
\text { underground }\end{array}$ & $\begin{array}{c}\text { Малоосвоенный район, } 45 \text { км до п. Аякс } \\
\text { временные дороги } \\
\text { Poorly developed area, } 45 \text { km to Ayax, } \\
\text { temporary roads }\end{array}$ & $1-24$ \\
\hline \multirow{2}{*}{$\begin{array}{l}\Pi 2 \\
\mathrm{P} 2\end{array}$} & $\begin{array}{c}\text { Хуличанское месторождение каменного угля } \\
\text { Khulichanskoe hard coal deposit }\end{array}$ & $\begin{array}{l}\text { Открытый } \\
\text { Surface }\end{array}$ & $\begin{array}{c}\text { Территория не освоена, расстояние по } \\
\text { автозимнику до п. Омолон } 75 \text { км } \\
\text { Undeveloped territory, winter road to the } \\
\text { village of Omolon }-75 \mathrm{~km}\end{array}$ & $3-4$ \\
\hline & $\begin{array}{c}\text { Верхнекегалинское } \\
\text { месторождение каменного угля } \\
\text { Verkhnekegalinskoe hard coal deposit }\end{array}$ & $\begin{array}{l}\text { Открытый } \\
\text { Surface }\end{array}$ & $\begin{array}{c}\text { Территория не освоена, } 70 \text { км до п. Кегали, } \\
\text { дороги отсутствуют } \\
\text { Undeveloped territory, } 70 \mathrm{~km} \text { to the village of } \\
\text { Kegali, no roads }\end{array}$ & $3-4$ \\
\hline \multirow[t]{2}{*}{$\begin{array}{l}\text { П3 } \\
\text { P3 }\end{array}$} & $\begin{array}{c}\text { Чайбухинское месторождение бурого угля } \\
\text { Chaybuhinskoe brown coal deposit }\end{array}$ & $\begin{array}{c}\text { Открытый } \\
\text { и подземный } \\
\text { Surface and } \\
\text { underground }\end{array}$ & $\begin{array}{c}\text { Слабоосвоенный район, } 100 \text { км до } \\
\text { п. Эвенск, временные дороги } \\
\text { Weakly developed area, } 100 \mathrm{~km} \text { to Evensk } \\
\text { settlement, temporary roads }\end{array}$ & $0,5-6$ \\
\hline & $\begin{array}{l}\text { Привозной каменный уголь } \\
\text { Hard coal from another region }\end{array}$ & - & - & - \\
\hline
\end{tabular}

Таблица 2. Характеристика рассматриваемых вариантов угля

Table 2. Characteristics of coal variants under consideration

\begin{tabular}{|c|c|c|c|c|c|c|c|}
\hline \multirow[t]{2}{*}{$\begin{array}{c}\text { Месторождение угля, } \\
\text { Coal deposit }\end{array}$} & \multirow[t]{2}{*}{$\begin{array}{l}\text { Марка } \\
\text { Tyре }\end{array}$} & \multicolumn{2}{|c|}{\begin{tabular}{|} 
Запасы категории, \\
млн т \\
Reserves by category, \\
mln tons
\end{tabular}} & \multirow{2}{*}{\begin{tabular}{|c|} 
Низшая теплота \\
сгорания на ра- \\
бочее состояние \\
$\mathrm{Q}_{i}^{r}$, ккал/кг \\
Net calorific value \\
$\mathrm{Q}_{i}^{r}, \mathrm{kcal} / \mathrm{kg}$ \\
\end{tabular}} & \multirow{2}{*}{$\begin{array}{c}\text { Зольность на } \\
\text { сухое состояние } \\
A^{d}, \% \\
\text { Ash per dry mass } \\
A^{d}, \% \\
\end{array}$} & \multirow{2}{*}{$\begin{array}{c}\text { Содержание азота на } \\
\text { сухое беззольное } \\
\text { состояние } N^{\text {daf }}, \% \\
\text { Nitrogen per dry ash- } \\
\text { free mass } N^{d a f}, \%\end{array}$} & \multirow{2}{*}{$\begin{array}{c}\text { Содержание серы } \\
\text { на сухое беззольное } \\
\text { состояние } S^{\text {daf }}, \% \\
\text { Sulfur per dry ash- } \\
\text { free mass } S^{\text {daf }}, \%\end{array}$} \\
\hline & & $\mathrm{A}+\mathrm{B}+\mathrm{C}_{1}$ & $\mathrm{P}_{1}+\mathrm{P}_{2}+\mathrm{P}_{3}$ & & & & \\
\hline Галимовское & $2 \mathrm{~A}$ & 157 & - & $\underline{4637-5920}$ & $11,6-30,0$ & $\underline{1,1-2,0}$ & $\underline{0,3-1,0}$ \\
\hline Galir & $2 \mathrm{~A}$ & $15, /$ & - & 5492 & & 1,5 & 0,5 \\
\hline $\begin{array}{l}\text { Кэновское } \\
\text { Kenovskoe }\end{array}$ & $\begin{array}{l}3 \mathrm{~A} \\
3 \mathrm{~A}\end{array}$ & 13 & - & $\frac{5521-6119}{5700}$ & $\frac{2,5-29,4}{16,8}$ & $\frac{0,9-1,3}{1,1}$ & $\frac{0,4-0,6}{0,5}$ \\
\hline $\begin{array}{c}\text { Булурское } \\
\text { Bulurskoe }\end{array}$ & $\begin{array}{l}2 \mathrm{~A} \\
2 \mathrm{~A}\end{array}$ & - & $39,6^{*}$ & $\frac{6226-7051}{6648}$ & $\frac{6,6-30,0}{13,8}$ & $\frac{0,5-1,7}{1,2}$ & $\frac{0,1-0,8}{0,4}$ \\
\hline $\begin{array}{c}\text { Хуличанское } \\
\text { Khulichanskoe }\end{array}$ & $\begin{array}{l}\text { Д } \\
\mathrm{D}\end{array}$ & - & $364 *$ & $\frac{3681-3963}{3817}$ & $\frac{12,3-30,8}{23,6}$ & $\frac{0,6-1,8}{1,4}$ & $\frac{0,2-0,6}{0,3}$ \\
\hline $\begin{array}{l}\text { Верхнекегалинское } \\
\text { Verkhnekegalinskoe }\end{array}$ & $\begin{array}{l}\text { ДГ } \\
\text { DG }\end{array}$ & - & $1540^{*}$ & $\frac{4111-5186}{4661}$ & $\frac{16,3-24,5}{19,7}$ & $\frac{0,5-1,9}{1,4}$ & $\frac{0,2-0,8}{0,4}$ \\
\hline $\begin{array}{c}\text { Чайбухинское } \\
\text { Chaybuhinskoe } \\
\end{array}$ & $\begin{array}{l}\mathrm{B} \\
\mathrm{B} \\
\end{array}$ & 6,3 & 40,8 & $\frac{2624-3652}{3152}$ & $\frac{6,5-48,9}{18,6}$ & $\frac{1,1-2,1}{1,5}$ & $\frac{0,5-1,4}{0,9}$ \\
\hline $\begin{array}{l}\text { Привозной уголь } \\
\text { Coal from another } \\
\text { region }\end{array}$ & $\begin{array}{l}\text { Д } \\
\mathrm{D}\end{array}$ & - & - & $\frac{4661-6310}{5449}$ & $\frac{12,1-24,0}{15,6}$ & $\frac{2,1-2,4}{2,2}$ & $\frac{0,4-0,7}{0,6}$ \\
\hline
\end{tabular}

* - пригодные для открытой добычи/suitable for surface mining.

Набор целей и критериев в задачах выбора топлива для ТЭС может варьироваться. Различие в критериях при сравнении угля в пунктах П1-ПЗ вызвано следующими причинами:

1. В пунктах П1 и П2 проводится сравнение только местных месторождений каменного угля, которые отличаются запасами и условиями освоения.

2. В пункте П3 бурый уголь местного месторождения сопоставляется с привозным каменным углем. Вводятся критерии оценки социального воздействия и воздействия выбросов на здоровье людей.
На втором этапе методики (рис. 2) проводится оценка альтернатив по критериям. При оценке угля по критерию $f_{1}$ «Удельная стоимость угля в условном эквиваленте» применяется формула:

$$
y_{1}=\frac{p_{c} k+p_{t} l}{Q_{i}^{r}} Q_{c e}
$$

где $p_{c}$ - цена угля, тыс. $\mathrm{p} / \mathrm{T} ; k$ - коэффициент, учитывающий условия добычи угля, о.е.; $p_{t}-$ удельная стоимость доставки тонны угля, тыс. $\mathrm{p} /$ км; $l$ - расстояние 
транспортировки угля, км; $Q_{c e}-$ теплотворная способность условного топлива, ккал/кг.

Используются следующие условия и допущения: $p_{c}$ для каменного угля принята в диапазоне 3,5-4 тыс. p/т; бурого - 2,5-3 тыс. p./т; $p_{t}$ принята в диапазоне от 0,017 до 0,019 тыс. p/км; $k=1$ при открытой добыче и 1,35 при подземной добыче, в соответствии со средними данными по отрасли. Расчет $y_{1}$ проводится для трех значений низшей теплоты сгорания (табл. 2).

Оценка альтернатив по критерию $f_{2}$ «Удельный ущерб от выбросов при сжигании топлива на ТЭС» проводится в соответствии с РД 34.02.305-98 «Методика определения валовых выбросов загрязняющих веществ в атмосферу от котельных установок ТЭС». Применяется выражение:

$$
y_{2}=M_{a} p_{a}+M_{\mathrm{NO}_{2}} p_{\mathrm{NO}_{2}}+M_{\mathrm{SO}_{2}} p_{\mathrm{SO}_{2}}+M_{\mathrm{CO}} p_{\mathrm{CO}},
$$

где $M_{a}, M_{\mathrm{NO}_{2}}, M_{\mathrm{SO}_{2}}, M_{\mathrm{CO}}$ - выбросы золы, оксидов азота, оксидов серы, оксида углерода, соответственно, т/т у.т.; $p_{a}, p_{\mathrm{NO}_{2}}, p_{\mathrm{SO}_{2}}, p_{\mathrm{CO}}-$ ставка платы за выбросы золы, оксидов азота, оксидов сера, оксида углерода, соответственно, $\mathrm{p} / \mathrm{T}$.

При расчете используются граничные и средние приведенные к рабочей массе значения содержания золы, азота и серы, а также три значения низшей теплоты сгорания угля (табл. 2).

Оценка угля по критерию $f_{31}$ проводится:

$$
y_{31}=\frac{\sum R_{t} k_{t}}{Q_{c e}} Q_{i}^{r},
$$

где $R_{t}$ - запасы угля $t$ категории, млн т; $k_{t}-$ коэффициент приведения прогнозных ресурсов угля к разведанным запасам [19].

При оценке угля по критерию $f_{41}$ используется шкала с тремя показателями (табл. 3), по которым экспертом назначаются баллы от 1 до 3. Больший балл назначается альтернативе с лучшей оценкой.

Таблица 3. Шкала для оценки месторождений по условиям освоения и добычи, балл

\begin{tabular}{|c|c|c|c|c|}
\hline $\begin{array}{c}\text { Месторождение, угленосный район } \\
\text { Coal deposit, coal-bearing region }\end{array}$ & $\begin{array}{c}\text { Способ освоения } \\
\text { Development method }\end{array}$ & $\begin{array}{c}\text { Близость населенных пунктов и дорог } \\
\text { Proximity of settlements and roads }\end{array}$ & $\begin{array}{c}\text { Мощность пластов угля } \\
\text { Coal steam thickness } \\
\end{array}$ & $\begin{array}{r}\text { Итого } \\
\text { Total } \\
\end{array}$ \\
\hline Галимовское/Galimovskoe & \begin{tabular}{|c|}
1 \\
\end{tabular} & 3 & 2 & 6 \\
\hline Кэновское/Kenovskoe & 3 & 3 & 1 & 7 \\
\hline Булурское/Bulurskoe & 2 & 2 & 3 & 7 \\
\hline Хуличанское/Khulichanskoe & 2 & 2 & 1 & 5 \\
\hline Верхнекегалинское/Verkhnekegalinskoe & 1 & 1 & 1 & 3 \\
\hline
\end{tabular}

Table 3. Scale for assessing coal deposits according to the conditions of development and mining, score

Критерий $f_{32}$ отличается сложностью получения достоверных значений. Преимуществом метода АНР является возможность сопоставления альтернатив с помощью оценок сравнительной предпочтительности $d_{i j}$. При этом может использоваться вспомогательная экспертная или статистическая информация. В частности, при сравнении воздействий выбросов бурого и каменного угля используются результаты исследований количества смертей на 1 ТВт·ч производства энергии на ТЭС [20] - 32,7 и 24,6 соответственно.

При оценке по критерию $f_{42}$ используются интервальные оценки шкалы сравнительной предпочтительности $d_{i j}$ в связи со сложностью определения количественных значений занятости местного населения. Во внимание принимается большая ожидаемая занятость местного населения при освоении местного
Чайбухинского месторождения в сравнении с использованием привозного топлива.

В табл. 4 представлены оценки альтернатив по критериям.

На третьем этапе выбора топлива (рис. 2) проводится построение однокритериальных интервальных функций ценности и определение ценности альтернатив по критериальным оценкам табл. 4.

На четвертом этапе проводится формирование матриц парных сравнений видов угля по формулам, аналогичным (8), (9) для определения минимальных, средних и максимальных частных ценностей альтернатив по каждому критерию.

На пятом этапе проводится оценка весовых коэффициентов критериев. В табл. 5 представлены результаты определения по формуле (3) весовых коэффициентов для двух вариантов предпочтений - ЛПР1 и ЛПР2.

Таблица 4. Оченки альтернатив по критериям

\begin{tabular}{|c|c|c|c|c|c|c|c|c|c|c|c|c|c|c|c|}
\hline \multirow{3}{*}{$\begin{array}{l}\text { Пункт } \\
\text { Point }\end{array}$} & \multirow{3}{*}{$\begin{array}{c}\text { Месторождение угля, } \\
\text { Coal deposit }\end{array}$} & \multicolumn{14}{|c|}{ Критерий/Criterion } \\
\hline & & \multicolumn{3}{|c|}{$\begin{array}{c}f_{1}, \text { тыс. } \mathrm{p} / \mathrm{\text {т }} \text { у.т. } \\
\text { thousand rubles/t c.e. }\end{array}$} & \multicolumn{3}{|c|}{$\begin{array}{l}f_{2}, \text { p/т у.т. } \\
\text { rubles/t c.e. }\end{array}$} & \multicolumn{3}{|c|}{$\begin{array}{c}f_{31}, \text { млн т у.т. } \\
\text { million t c.e. }\end{array}$} & \multirow{2}{*}{ 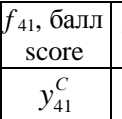 } & \multicolumn{2}{|c|}{$\begin{array}{c}f_{32}, \text { балл } \\
\text { score }\end{array}$} & \multicolumn{2}{|c|}{$\begin{array}{l}f_{42}, \text { балл } \\
\text { score }\end{array}$} \\
\hline & & $y_{1}^{L}$ & $y_{1}^{C}$ & $y_{1}^{R}$ & $y_{2}^{L}$ & $y_{2}^{C}$ & $y_{2}^{R}$ & $y_{31}^{L}$ & $y_{31}^{C}$ & $y_{31}^{R}$ & & $y_{32}^{L}$ & $y_{32}^{R}$ & $y_{42}^{L}$ & $y_{42}^{R}$ \\
\hline \multirow{3}{*}{$\begin{array}{l}\Pi 1 \\
\text { Р1 }\end{array}$} & Галимовское/Galimovskoe & 6,0 & 6,9 & 8,7 & 0,9 & 1,4 & 2,5 & 10,4 & 12,3 & 13,3 & 6 & \multicolumn{2}{|r|}{-} & \multicolumn{2}{|c|}{-} \\
\hline & Кэновское/Kenovskoe & 4,8 & 5,5 & 6,0 & 0,7 & 0,9 & 1,2 & 10,3 & 10,6 & 11,4 & 7 & \multicolumn{2}{|c|}{-} & \multicolumn{2}{|c|}{-} \\
\hline & Булурское/Bulurskoe & 5,5 & 6,2 & 7,0 & 0,4 & 0,8 & 1,5 & 12,3 & 13,2 & 19,9 & 7 & \multirow{2}{*}{\multicolumn{2}{|c|}{-}} & \multicolumn{2}{|c|}{-} \\
\hline \multirow[b]{2}{*}{$\begin{array}{l}\text { П2 } \\
\text { Р2 }\end{array}$} & Хуличанское/Khulichanskoe & 11,0 & 12,1 & 13,3 & 0,8 & 1,5 & 2,1 & 18,1 & 18,8 & 26,4 & 5 & & & & - \\
\hline & \begin{tabular}{|l|} 
Верхнекегалинское \\
Verkhnekegalinskoe \\
\end{tabular} & 9,8 & 11,6 & 14,0 & 0,7 & 1,4 & 2,3 & 63,4 & 71,9 & 114 & 3 & \multicolumn{2}{|c|}{-} & \multicolumn{2}{|c|}{-} \\
\hline \multirow{2}{*}{$\begin{array}{l}\Pi 3 \\
\text { Р3 }\end{array}$} & Чайбухинское/Chaybuhinskoe & 8,2 & 10,3 & 13,3 & 1,4 & 2,4 & 4,4 & - & - & - & - & 4 & 6 & 4 & 6 \\
\hline & $\begin{array}{l}\text { Привозной уголь } \\
\text { Coal from another region }\end{array}$ & 11,1 & 13,5 & 16,5 & 1,3 & 1,8 & 2,4 & - & - & - & - & -4 & -6 & -4 & -6 \\
\hline
\end{tabular}

Table 4. Assessments of alternatives by criteria 
Таблица 5. Оиенки весовых коэффициентов критериев

Table 5. Assessments of criteria weights

\begin{tabular}{|c|c|c|c|c|c|c|c|}
\hline Пункт/Point & Пункт/Point & $f_{1}$ & $f_{2}$ & $f_{31}$ & $f_{41}$ & $f_{32}$ & $f_{42}$ \\
\hline П1, П2 & ЛПР1/DM1 & 0,400 & 0,100 & 0,100 & 0,400 & - & - \\
\cline { 2 - 8 } P1, P2 & ЛПР2/DM2 & 0,250 & 0,125 & 0,125 & 0,500 & - & - \\
\hline П3 & ЛПР1/DM1 & 0,615 & 0,154 & - & - & 0,077 & 0,154 \\
\cline { 2 - 8 } P3 & ЛПP2/DM2 & 0,500 & 0,250 & - & - & 0,125 & 0,125 \\
\hline
\end{tabular}

В рассматриваемом примере при выборе угля в пунктах П1, П2 большее значение для ЛПР имеют критерии удельной стоимости топлива и условий освоения и добычи, а в пункте ПЗ большее значение имеет критерий удельной стоимости топлива. Два варианта предпочтений ЛПР отличаются не только значениями весовых коэффициентов, но и их ранжированием.

На шестом этапе проводится многокритериальная оценка вариантов топлива для ТЭС. На рис. 5 вертикальные линии характеризуют диапазон многокритериальных оценок каждой альтернативы, прямоугольники отражают средние многокритериальные оценки для двух вариантов предпочтений ЛПР.

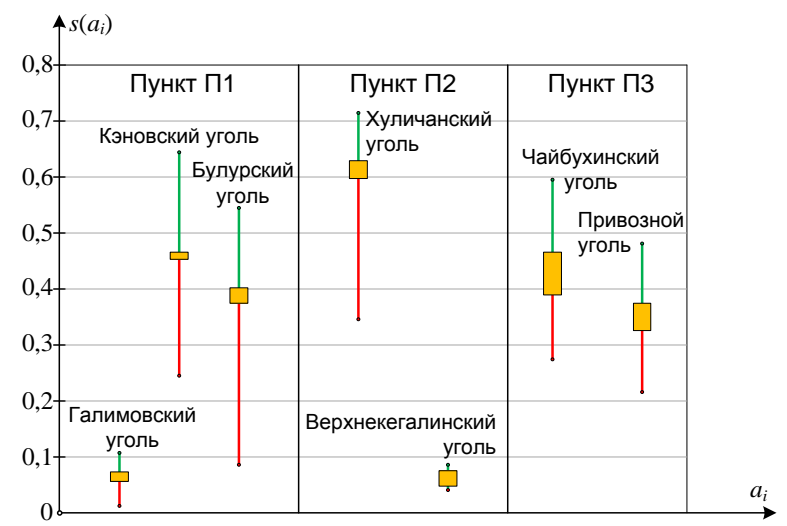

Pис. 5. Многокритериальные оченки угля в пунктах П1, $\Pi 2, \Pi 3$

Fig. 5. Multi-criteria coal estimates in points $P 1, P 2, P 3$

В пункте П1 угли Кэновского и Булурского месторождений имеют более высокие многокритериальные оценки по сравнению с оценками угля Галимовского месторождения. Это соответствует соотношению оценок альтернатив по критериям с наибольшим весом $-f_{1}$ и $f_{41}$ (табл. 4,5$)$.

В пункте П2 многокритериальные оценки угля Хуличанского месторождения существенно превышают оценки угля Верхнекегалинского месторождения из-за большого отличия по критерию $f_{41}-$ условия освоения и добычи.

В пункте ПЗ альтернативы имеют сопоставимые многокритериальные оценки с небольшим превосходством угля Чайбухинского месторождения.

На седьмом этапе (рис. 3) проводится ранжирование альтернатив. В пункте П2 выбор альтернативы однозначен - уголь Хуличанского угленосного района в большей степени соответствует предпочтениям ЛПР (рис. 5). В пунктах П1 и ПЗ для ранжирования альтернатив необходимо использовать модели сравнения (10), (11). При сравнении углей Кэнского и Булурского месторождений получены результаты:
$F_{\text {domij }}=0,71 ; \quad F_{s i j}=0,71$. В результате сопоставления Чайбухинского и привозного угля получены оценки $F_{\text {domij }}=0,67 ; F_{s i}=0,74$. Оценки $F_{\text {donij }}, F_{s i j}$ позволяют сделать вывод, что в пункте П2 при высказанных предпочтениях ЛПР уголь Кэновского месторождения более предпочтителен, в пункте ПЗ более предпочтителен уголь Чайбухинского месторождения.

Полученные результаты выбора угля согласуются с оценками альтернатив по критериям и структурой предпочтений ЛПР. Ранжирование альтернатив устойчиво к изменениям исходной информации и предпочтений ЛПР в пределах заданных интервалов.

\section{Выводы}

1. Реформирование энергетики привело к трансформации методологии обоснования ее развития от централизованного государственного планирования к новой парадигме многостороннего процесса обоснования решений в условиях многокритериальности, множественности несовпадающих интересов, неопределенности исходной информации и условий дальнейшего развития. В результате выбор топлива для тепловых электростанций в районах нового освоения в современных условиях представляет собой многокритериальную проблему с неопределенностью исходной информации и неоднозначностью предпочтений лиц, принимающих решения.

2. Ввиду тенденций повышения значимости экологических и социальных факторов выбор топлива для тепловых электростанций целесообразно проводить с использованием многокритериальных методов рационального выбора. Широко распространенным является метод анализа иерархий, что объясняется такими преимуществами, как удобство применения для лица, принимающего решения, специальная шкала для сравнения альтернатив по сложно формализуемым критериям. Для использования метода анализа иерархий в условиях неопределенности исходной информации и предпочтений лиц, принимающих решения, предложена модификация метода. Для ранжирования альтернатив в условиях неопределенности предложены две модели оценивания: на основе относительного доминирования, а также на основе суммы разностей многокритериальных оценок.

3. Предложена методика многокритериального выбора топлива на основе модифицированного метода мультипликативного анализа иерархий. Ее применение рассмотрено на проблеме выбора угля для тепловой электростанции в трех пунктах перспективного размещения Северо-Эвенского и Омсукчанского районов Магаданской области. 
Для сравнения топлива разработана иерархия целей и критериев, отражающая экономические, технические, экологические и социальные факторы. При принятии решений была учтена неопределенность исходной информации, неоднозначность предпочтений лиц, принимающих решения. Формализация проблемы выбора топлива позволяет получить количественные оценки альтернативных вариантов для принятия окончательного

\section{СПИСОК ЛИТЕРАТУРЫ}

1. Системные исследования в энергетике: ретроспектива научных направлений Сибирского энергетического института Института систем энергетики им. Л.А. Мелентьева / отв. ред. Н.И. Воропай. - Новосибирск: Наука, 2010. - 686 с.

2. Life cycle assessment of electricity generation: a review of the characteristics of existing literature / M.V. Barros, R. Salvador C.M. Piekarski, A.C. de Francisco, F.M.C.S. Freire // International Journal of Life Cycle Assessment. - 2020. - V. 25 (1). - P. 36-54.

3. A multi-criteria analysis of coal-based power generation in Bangladesh / R. Zaman, Th. Brudermann, S. Kumar, N. Islam // Energy Policy. - 2018. - V. 116. - P. 182-192.

4. More priorities, more problems? Decision-making with multiple energy, development and climate objectives / A. Bhardwaj, M. Joshi, R. Khosla, N.K. Dubash // Energy Research and Social Science. - 2019. - V. 49. - P. 143-157.

5. Multi-criteria analysis of electricity generation scenarios for sustainable energy planning in Pakistan / M.N. Hussain, M.A. Uqaili, K. Harijan, M.W. Mustafa, M.M. Rahman, M.W.A. Khan // Energies. - 2018. - V. 11. - Iss. 3. - 757 p.

6. Экономико-математические методы и модели принятия решений в энергетике / Артюгина И.М., Вастл Я., Воронкин А.Ф., Габржинский И., Гусев В.Н., Долгов П.П., Дудоркин И., Ирешова А., И. Клима, Э.М. Косматов, Т.В. Лисочкина, В.Р. Окороков, Р. Повышел, В. Скуровец, В.А. Таратин / под ред. П.П. Долгова, И. Климы. - Л.: Изд-во Ленинградского университета, 1991. - $224 \mathrm{c}$

7. Selection ideal coal suppliers of thermal power plants using the matter-element extension model with integrated empowermen method for sustainability / T. Zhongfu, J. Liwei, Y. Xiaobao, Zh. Huijuan, Yu. Chao // Mathematical Problems in Engineering. - 2014. - V. 2014. - 302748.

8. Yucekaya A. Multi-objective fuel supply for coal-fired power plants under emission, transportation and operational constraints // Energy Sources, Part B: Economics, Planning and Policy. 2013. - V. 8:2. - P. 179-189.

9. Durairaj S., Sathiyasekar K., Ilangkumaran M. Selection of alternate fuel for electrical power generator using hybrid multi criteria decision making technique // Scientific Bulletin-University решения. Предложенная методика может использоваться на стадии предпроектных исследований для выявления наиболее перспективных вариантов топливообеспечения тепловых электростанций в районах нового освоения.

Работа выполнена в рамках проекта государственного задания 17.6 .1 (рег. № АAAA-A17-117030310445-9) фундаментальных исследований СО РАН.

Politehnica of Bucharest, Series C. - 2016. - V. 78. - Iss. 1. P. $247-258$

10. Dong F. Coal suppliers evaluation model based on AHP and PROMETHEE // 2nd International Conference on Education Reform and Modern Management (ERMM 2015). - Hong Kong, 2015. - P. 359-362.

11. Triantaphyllou E. Two new cases of rank reversals when the AHP and some of its additive variants are used that do not occur with the multiplicative AHP // Journal of Multi-Criteria Decision Analysis. - 2001. - V. 10. - P. 11-25.

12. Lootsma F.A. Scale sensitivity in the multiplicative AHP and SMART // Journal of Multi-Criteria Decision Analysis. - 1993. V. 2. - P. 87-110.

13. Buckley J.J. Fuzzy hierarchical analysis // Fuzzy Sets and Systems. - 1985. - V. 17. - P. 233-247.

14. Chang D. Applications of the extent analysis method on fuzzy AHP // European Journal of Operational Research. - 1996. V. 95. - P. 649-655.

15. Шакиров В.А. Многокритериальная оценка альтернатив на основе теории полезности в условиях неопределенности предпочтений лица, принимающего решения // Нечеткие системы и мягкие вычисления. - 2018. - Т. 13. - № 1. - С. 17-35.

16. Геология месторождений угля и горючих сланцев СССР. Т. 10. Угольные бассейны и месторождения Северо-Востока СССР и Камчатки. - М.: Госгеолтехиздат, 1962. - 403 с.

17. Угольная база России. Т. V. Кн. 2. Угольные бассейны и месторождения Дальнего Востока России (Республика Саха, Ceверо-Восток, о. Сахалин, п-ов Камчатка). - М.: ЗАО «Геоинформмарк», 1999. - $638 \mathrm{c}$

18. Минерально-сырьевая база угольной промышленности России. В 2-х т. Т. 2 (регионы и бассейны). - М.: Изд-во Московского государственного горного университета, 1999. - 448 с.

19. Неженский И.А., Павлова И.Г. Методические основы оценки стоимости российских недр // Минеральные ресурсы России. Экономика и управление. - 1995. - № 4. - С. 13-18.

20. Маремуха Т.П., Петросян А.А. Загрязнение атмосферного воздуха фракциями мелкодисперсной пыли (PM10 и РМ2,5) в районе функционирования угольной ТЭС // Здоровье и окружающая среда. - 2016. - № 26. - С. 39-42.

Поступила 02.09.2020 г.

\section{Информация об авторах}

Шакиров В.А., кандидат технических наук, старший научный сотрудник отдела комплексных и региональных проблем энергетики Института систем энергетики им. Л.А. Мелентьева СО РАН. 
UDC 620.98:519.816

\title{
CHOICE OF FUEL FOR HEAT POWER PLANTS IN AREAS OF NEW DEVELOPMENT TAKING INTO ACCOUNT THE UNCERTAINTY FACTOR
}

\author{
Vladislav A. Shakirov, \\ mynovember@mail.ru \\ Melentiev Energy systems institute SB RAS, \\ 130, Lermontov street, Irkutsk, 664033, Russia.
}

The relevance of the research is caused by the transformation of the methodology for substantiating the development of energy from centralized state planning to a new paradigm for multilateral decision-making and the creation of mechanisms for their implementation in terms of multi-criteria, multiplicity of conflicting interests, uncertainty of initial information and conditions for further development. New conditions for substantiating the decisions are considered in the context of the construction of thermal power plants in remote areas of new development, where local energy resources are considered as fuel. The choice of fuel determines the economic and technical indicators of power plants, their environmental and social impacts. In order to justify the choice of fuel for thermal power plants from the point of view of numerous criteria in the conditions of uncertainty of initial information and future development conditions, it is necessary to create new methodological approaches.

The main aim of the research is to propose a methodology of multi-criteria fuel selection for a thermal power plant in the areas of new development taking into account the uncertainty factor.

Objects: organic fuel deposits in remote areas of new development.

Methods: multiplicative method of the analytic hierarchy process, method of multi-criteria utility theory, methods of interval analysis, methods of fuzzy set theory.

Results. The author has carried out the review of modern methodological approaches in the problems of substantiation of the choice of fuel for power plants and proposed the modification of the method of the analytic hierarchy process. It allows taking into account the uncertainty of the source information and the ambiguity of preferences of decision makers, as well as significantly reducing the number of requests for information. On the basis of the modified method, the author developed the technique of multi-criteria selection of fuel for thermal power plants in the areas of new development. Its application is considered on the problem of coal selection in three points of perspective siting of thermal power plant in Omsukchansky and Severo-Evensky districts of Magadan region. The criteria used to compare the alternatives are: fuel cost, pollutant emissions from fuel combustion, fuel reserves, development and production conditions, the impact of fuel combustion emissions on the health of the population, employment of the local population.

\section{Key words:}

Thermal power plants, fuel, coal, multi-criteria choice, uncertainty of the initial information.

The research was carried out under the State Assignment Project 17.6 .1 (reg. number AAAA-A17-117030310445-9) of the Fundamental Research of the Siberian Branch of the Russian Academy of Sciences.

\section{REFERENCES}

1. Sistemnye issledovaniya $v$ energetike: retrospektiva nauchnykh napravlenii Sibirskogo energeticheskogo instituta-Instituta sistem energetiki im. L.A. Melenteva [Systems research in the energy sector: retrospective scientific directions of Siberian energy institute - Melentiev energy systems institute]. Ed. by N.I. Voropai. Novosibirsk, Nauka Publ., 2010. 686 p.

2. Barros M.V., Salvador R., Piekarski C.M., de Francisco A.C., Freire F.M.C.S. Life cycle assessment of electricity generation: a review of the characteristics of existing literature. International Journal of Life Cycle Assessment, 2020, vol. 25 (1), pp. 36-54

3. Zaman R., Brudermann Th., Kumar S., Islam N. A multi-criteria analysis of coal-based power generation in Bangladesh. Energy Policy, 2018, vol. 116, pp. 182-192.

4. Bhardwaj A., Joshi M., Khosla R., Dubash N.K. More priorities, more problems? Decision-making with multiple energy, development and climate objectives. Energy Research and Social Science, 2019, vol. 49, pp. 143-157.

5. Hussain Mirjat N., Uqaili M.A., Harijan K., Mustafa M.W., Rahman M.M., Khan M.W.A. Multi-criteria analysis of electricity generation scenarios for sustainable energy planning in Pakistan. Energies, 2018, vol. 11, 757

6. Artyugina I.M., Vastl Ya., Voronkin A.F., Gabrzhinskii I., Gusev V.N., Dolgov P.P., Dudorkin I., Ireshova A., Klima I., Kosmatov E.M., Lisochkina T.V., Okorokov V.R., Povyshel R., Skurovets V., Taratin V.A. Ekonomiko-matematicheskie metody $i$ modeli prinyatiya reshenii $v$ energetike [Economic and mathematical methods and decision-making models in the energy sector]. Eds. P.P. Dolgov, I. Klima. Leningrad, Leningrad University Press, 1991. 224 p.

7. Zhongfu T., Liwei J., Xiaobao Y., Huijuan Zh., Chao Yu. Selection ideal coal suppliers of thermal power plants using the matter-element extension model with integrated empowerment method for sustainability. Mathematical Problems in Engineering, 2014, vol. 2014, 302748 .

8. Yucekaya A. Multi-objective fuel supply for coal-fired power plants under emission, transportation and operational constraints. Energy Sources, Part B: Economics, Planning and Policy, 2013, vol. 8:2, pp. 179-189.

9. Durairaj S., Sathiyasekar K., Ilangkumaran M. Selection of alternate fuel for electrical power generator using hybrid multi criteria decision making technique. Scientific Bulletin-University Politehnica of Bucharest, Series C, 2016, vol. 78, Iss. 1, pp. $247-258$.

10. Dong F. Coal suppliers evaluation model based on AHP and PROMETHEE. $2^{\text {nd }}$ International Conference on Education Reform and Modern Management (ERMM 2015). Hong Kong, 2015. pp. 359-362.

11. Triantaphyllou E. Two new cases of rank reversals when the AHP and some of its additive variants are used that do not occur with the multiplicative AHP. Journal of Multi-Criteria Decision Analysis, 2001, vol. 10, pp. 11-25.

12. Lootsma F.A. Scale sensitivity in the multiplicative AHP and SMART. Journal of Multi-Criteria Decision Analysis, 1993, vol. 2, pp. $87-110$

13. Buckley J.J. Fuzzy hierarchical analysis. Fuzzy Sets and Systems, 1985, vol. 17, pp. 233-247. 
14. Chang D. Applications of the extent analysis method on fuzzy AHP. European Journal of Operational Research, 1996, vol. 95, pp. 649-655.

15. Shakirov V.A. Multi-criteria evaluation of alternatives under conditions of uncertainty in the decision maker's preferences based on utility theory. Nechetkie sistemy i myagkie vychisleniya, 2018, vol. 13, no. 1, pp. 17-35. In Rus.

16. Geologiya mestorozhdenii uglya i goryuchikh slantsev SSSR. T. 10. Ugolnye basseiny $i$ mestorozhdeniya Severo-Vostoka SSSR $i$ Kamchatki [Geology of deposits of coal and oil shale of the USSR. Vol. 10. Coal basins and deposits of the North-East of the USSR and Kamchatka]. Moscow, Gosgeoltekhizdat Publ., 1962. 403 p.

17. Ugolnaya baza Rossii. T. V. Kn. 2. Ugolnye basseiny mestorozhdeniya Dalnego Vostoka Rossii (Respublika Sakha, Severo-Vostok, o. Sakhalin, p-ov Kamchatka) [Coal base of Russia. Vol. 5. B. 2. Coal basins and deposits of the Russian Far
East (Republic of Sakha, Northeast, Sakhalin Island, Kamchatka Peninsula). Moscow, Geoinformmark Publ., 1999. 638 c.

18. Mineralno-syrevaya baza ugolnoi promyshlennosti Rossii. T. 2 (regiony i basseiny) [Mineral resources base of the coal industry of Russia. Vol. 2 (regions and basins)]. Moscow, Moscow State Mining University Publ. House, 1999. 448 p.

19. Nezhenskii I.A., Pavlova I.G. Metodicheskie osnovy otsenki stoimosti rossiiskikh nedr [Methodological basis for assessing the cost of Russian subsoil]. Mineral resources of Russia. Economics and management, 1995, no. 4, pp. 13-18.

20. Maremukha T.P., Petrosian A.A. Particulate matter air pollution $\left(\mathrm{PM}_{10}\right.$ and $\left.\mathrm{PM}_{2.5}\right)$ in the coal HPP area. Health and environment, 2016, no. 26, pp. 39-42. In Rus.

Received: 2 September 2020.

\section{Information about the authors}

Vladislav A. Shakirov, Cand. Sc., senior researcher, Melentiev energy systems institute SB RAS. 